\title{
The Emotion Regulation Questionnaire in Women with Cancer: A Psychometric Evaluation and an Item Response Theory Analysis
}

Tânia Brandão ${ }^{1}$, Marc S. Schulz ${ }^{2}$, James J. Gross ${ }^{3}, \&$ Paula Mena Matos ${ }^{1}$

${ }^{1}$ Faculty of Psychology and Education Sciences, University of Porto, Portugal; Center for Psychology at University of Porto.

${ }^{2}$ Department of Psychology, Bryn Mawr College, Pennsylvania, USA.

${ }^{3}$ Department of Psychology, Stanford University, USA.

Corresponding authors: Paula Mena Matos - Faculty of Psychology and Education Sciences, University of Porto, Portugal; Center for Psychology at University of Porto. Email: pmmatos@fpce.up.pt

Funding: PhD Scholarship from the Portuguese Foundation for Science and Technology granted to the first author (SFRH/BD/84436/2012).

Please Note: This is the author's version of the manuscript accepted for publication in PsychoOncology. Changes resulting from the publishing process, namely editorial editing, corrections, final formatting for publication, and other modifications resulting from quality control mechanisms may have been subsequently added.

A definitive version was subsequently published as: Brandão, T., Schulz, M. S., Gross, J. J, \& Matos, P. M. (2017). The Emotion Regulation Questionnaire in Women with Cancer: A Psychometric Evaluation and an Item Response Theory Analysis. Psycho-oncology, 26, 16471653. Doi: $10.1002 /$ pon. 4356 


\begin{abstract}
Objective: Emotion regulation is thought to play an important role in adaptation to cancer. However, the Emotion Regulation Questionnaire (ERQ), a widely-used instrument to assess emotion regulation, has not yet been validated in this context. This study addresses this gap by examining the psychometric properties of the ERQ in a sample of Portuguese women with cancer. Methods: The ERQ was administered to 204 women with cancer $(\mathrm{M}$ age $=48.89$ years, $\mathrm{SD}=$ 7.55). Confirmatory factor analysis (CFA) and item response theory (IRT) analysis were used to examine psychometric properties of the ERQ.

Results: CFA confirmed the two-factor solution proposed by the original authors (expressive suppression (ES) and cognitive reappraisal (CR)). This solution was invariant across age and type of cancer. IRT analyses showed that all items were moderately to highly discriminant and that items are better suited for identifying moderate levels of ES and CR. Support was found for the internal consistency and test-retest reliability of the ERQ. The pattern of relationships with emotional control, alexithymia, emotional self-efficacy, attachment, and quality of life provided evidence of the convergent and concurrent validity for both dimensions of the ERQ.
\end{abstract}

Conclusion: Overall, the ERQ is a psychometrically sound approach for assessing emotion regulation strategies in the oncological context. Clinical implications are discussed. 


\section{Introduction}

After being diagnosed with cancer, patients have to manage a wide range of emotions [1]. It is now clear that the way cancer patients regulate these emotions influences their psychological functioning and adaptation to cancer [e.g., 2,3]. Despite widespread agreement that it is essential to better understand how cancer patients manage their emotions, systematic study of this issue is complicated by disagreement regarding the definition and measurement of emotion regulation (ER) [see 4 for a discussion]. ER refers to attempts to influence which emotions one has, and how one experiences and expresses these emotions [5,6], and can involve modulation of any component of the emotion process, including appraisal of the situation that stimulates emotion and the varied channels of emotional response [6]. ER strategies can be characterized in terms of the degree of engagement with (e.g., talking about a fear) or distancing from (e.g., suppression) difficult emotional experiences [7]. ER strategies also differ depending on what stage of the emotion process they are primarily targeting [8].

One of the most widely used instruments for assessing ER is the Emotion Regulation Questionnaire (ERQ) [9], a self-report questionnaire assessing two ER strategies with 10 items. Cognitive reappraisal $(\mathrm{CR})$ assesses the tendency of individuals to alter an emotional response by reinterpreting the meaning of a specific stimulus. CR has been characterized as an antecedentfocused ER strategy since it is focused on the emotion generative process [10]. Expressive suppression (ES) assesses the tendency of individuals to inhibit or control behavioral expressions of an emotional response, and is considered a response-focused strategy since it addresses response tendencies that have been generated in an emotion context [10].

The ERQ has good psychometric properties in terms of factorial validity, internal consistency, test-retest reliability, and convergent and discriminant validity. Gross and John [9] 
found that the ERQ fit well into an independent, two-factor model (i.e., zero correlation between factors). The remaining models they examined all fit significantly worse. In the original psychometric studies [9], each of the two dimensions of the ERQ had adequate internal consistency across different samples as well as good test-retest reliability across 3 months. Evidence for convergent and discriminant validity was also obtained [see 9 for details].

Psychometric properties of the ERQ have been investigated in many populations ${ }^{1}$ [e.g., 11,12 ] and languages [e.g., 13; translations are available in more than 25 languages (see http://spl.stanford.edu/resources.html)]. Most studies have confirmed the two-factor structure although in some cases items did not load as expected $[11,14]$. Moreover, the independence of the two factors proposed by Gross and John [9] has not been uniformly supported. While some studies have found that an independent, two-factor model provided the best fit [e.g., 15,16], other studies found evidence supporting a correlated-factors model [e.g., 12, 17].

To our knowledge, there is no systematic study on the psychometric of the ERQ in the oncological context. The paucity of psychometric studies in this context may be contributing to its sparse use in studies of psychosocial adjustment to cancer. A recent review found no studies using the ERQ to assess ER strategies in women with breast cancer, one of the most commonly studied cancers regarding psychosocial outcomes [4].

This study aimed to examine the psychometric properties of the ERQ in a sample of women with cancer. Specifically, we aimed (1) to test the two-factorial structure of the ERQ proposed by Gross and John [9] using a CFA, and to examine ERQ scale reliability; (2) to identify the psychometric properties of each item, including patterns of responses to items and

${ }^{1}$ These studies included populations from the community (1033 participants; 731 females; aged 17-95; M= 39.1; SD = 20.5) and sport participants (433 athletes; 296 males; aged 18-47; M = 20.45; SD = 2.79). Other validation studies have been conducted, but a full review of these studies is beyond the scope of this study. 
differential item functioning across groups using an IRT analysis; and (3) to obtain evidence regarding convergent, concurrent, and discriminant validity.

\section{Methods}

\section{Participants}

Participants were recruited online between December 2015 and February 2016, through a web-based survey described in Facebook groups and pages related to cancer and in an online Portuguese platform called "Talk about Cancer." Criteria for inclusion were having a diagnosis of cancer and being more than 18 years old. The final sample included 204 women $(M=48.89$ years, $S D=7.55)$. Breast cancer $(67 \%)$ was by far the most common diagnosis (see supporting information Table S1). 148 women provided email information for follow-up contact, but only 44 women (30\%) completed the ERQ questionnaire again after a period of 6-weeks.

\section{Measures}

The Emotion Regulation Questionnaire (ERQ). The Portuguese translated version of the ERQ available from Gross's website was used. The ERQ [9] is a 10-item self-report questionnaire designed to assess two specific strategies of ER: ES (4 items) and CR (6 items), using a Likert-type scale ranging from 1 (strongly disagree) to 7 (strongly agree).

The Courtauld Emotional Control Scale (CECS). The CECS [18; Portuguese version: 19] is a 21-item self-report scale designed to assess the tendency to control or suppress the expression of negative emotions. Items are rated on a Likert-type scale ranging from 1 (almost never) to 4 (almost always). The CECS has three separate subscales assessing the suppression or 
expression of anger, anxiety, and depressed mood. Cronbach's $\alpha$ was .82 for anger, .84 for anxiety, .85 for depressed mood, and .92 for the total score.

The Stanford Emotional Self-Efficacy Scale - Cancer (SESES-C) The SESES-C [20; Portuguese version: 21] is a 15-item self-report scale designed to assess emotional self-efficacy in cancer patients. Items are rated on a Likert-type scale ranging from $0 \%$ (not at all confident) to 100\% (completely confident). The SESES-C has three subscales each containing five items:

communicating emotions, focusing on the present, and confronting death/dying issues. We used the first two subscales. Cronbach's $\alpha$ was .79 for communicating emotions, .78 for focusing on the present, and .81 for the total scale score.

The Toronto Alexithymia Scale - 20 (TAS-20). The TAS-20 [22; Portuguese version: 23 ] is a 20-item self-report questionnaire designed to assess alexithymia or difficulty in identifying and describing emotions. Items are rated on a Likert-type scale ranging from 1 (strongly disagree) to 5 (strongly agree). It comprises three subscales, namely difficulty in identifying the feelings (DIF) (7 items), difficulty in describing the feelings (DDF) (5 items) and focus on external experiences (FEE) ( 8 items). Cronbach's $\alpha$ was .83 for DIF, .65 for DDF, .43 for FEE, and .83 for the total score. Because FEE did not have adequate internal consistency, it was removed from further analyses.

\section{The Experiences in Close Relationships - Relationship Structures Questionnaire} (ECR-RS). The ECR-RS [24; Portuguese version: 25] is a 9-item self-report questionnaire designed to assess attachment anxiety ( 3 items) and attachment avoidance (6 items) in close relationships. Participants are asked to identify an adult with whom they have a close and strong emotional relationship; then they are asked to rate each item with respect to that person. Items 
are rated on a Likert-type scale ranging from 1 (strongly disagree) to 7 (strongly agree).

Cronbach's $\alpha$ was .91 for attachment anxiety and .82 for attachment avoidance.

The World Health Organization Quality of Life - BREF (WHOQOL-BREF). The WHOQOL-BREF [26] is a 26-item self-report questionnaire designed to measure four specific domains of QOL: physical health (7 items), psychological (6 items), social (3 items), and environment ( 8 items). We examined QOL in three domains: physical health $(\alpha=.85)$, psychological $(\alpha=.82)$, and social $(\alpha=.67)$.

Demographic information. Sociodemographic (e.g., age) and clinical information (e.g., site of cancer) were also collected.

\section{Procedure}

The study was approved by the Ethics Committee of the Faculty of Psychology and Educational Sciences, University of Porto. Data were collected online using LimeSurvey. Participants were not approached directly by researchers but were invited to participate in the study through a post in Facebook pages and groups related to cancer issues and in an online platform called "Talk about Cancer". The time required to complete the questionnaires varied (15-25 min). Participants were volunteers and did not receive any type of compensation for their participation. To examine test-retest reliability, 6-weeks following the initial questionnaire an invitation to complete the ERQ again was sent to those participants who voluntarily left their email for further contact. 


\section{Statistical Analysis}

Since answers to items were marked as required (i.e., participants had to provide an answer before they could proceed to the next question) there were no missing data. CFA was performed using structural equation modeling software (AMOS). IRT analysis was carried out using IRTPRO [27].

\section{Results}

\section{Preliminary Analyses}

Descriptive analyses for each item and for the two subscales are presented in supporting information Table S2. Skewness $(<.2)$ and kurtosis $(<.7)$ values indicate no serious departures from normality [28].

\section{Structural Validity and Reliability}

CFA using maximum likelihood estimation was conducted to test the two-factor structure of the ERQ. Goodness-of-fit indicators used were: the chi-square/df statistic $(<2.0)$, the Bentler comparative fit index (CFI) and the goodness of fit index (GFI) (>.95), the standardized root mean square residual (SRMR; <.06) and the root mean square error of approximation (RMSEA; $<.07)$ [29]. Multi-group analyses were performed to test invariance of the ERQ across groups (age and type of cancer).

We followed the procedures used by Gross and John [9] and tested five possible models: an unconstrained model (two factors correlating freely), a general factor model (all 10 items loading on a general factor), a hierarchical model (two factors correlating .50), a specialist model 
(two factors correlating -.50), and an independent model (two factors correlating zero). Model fit for models are presented in supporting information Table S3. Only the unconstrained model presented a good fit to the data across all indicators $\left(\chi^{2}(33)=59.71 ; p=.003 ; \chi^{2} / \mathrm{df}=1.81 ; \mathrm{CFI}=.96\right.$; $\mathrm{GFI}=.95 ; \mathrm{SRMR}=.05 ; \mathrm{RMSEA}=.06,90 \% \mathrm{CI}(.036, .088), \mathrm{pclose}<.05)$.

The final CFA for the unconstrained model is displayed in Figure 1. The results confirmed the two-factor structure (all items loading significantly on the expected factor with loadings equal to or greater than .40). Model modification indices suggested the inclusion of correlated errors between item 1 and item 3 . The inclusion of correlated errors is justified by the items' semantic similarity, and is similar to the approach taken in recent studies [11, 30]. The correlation between the ES and CR factors was .33, indicating some overlap but much independence among the two factors.

Multi-group analyses to test structural invariance according to age and type of cancer were performed. The sample was divided into younger women $(\mathrm{n}=111 ; 24-50$ years $)$ and older women ( $\mathrm{n}=93 ; 51-66$ years) reflecting the understanding that women under 50 years age are considered to have cancer at a "young age" since they are premenopausal [31]. The sample was also divided into those who had breast cancer and those who had other types of cancer. Analyses provided evidence for measurement invariance across age and type of cancer (see supporting information Table S4).

Reliability of the ERQ was assessed using internal consistency (Cronbach's alpha coefficients; >.70) and test-retest reliability (intra-class correlation coefficient (ICC); > 40) [32]. Both subscales demonstrated adequate internal consistency $(\alpha=.72$ and .82 for ES and CG, respectively) and good test-retest stability $(\mathrm{ES} \mathrm{ICC}=.70,95 \% \mathrm{CI}(.508, .823), p<.001$; CR $\mathrm{ICC}=.51,95 \% \mathrm{CI}(.260, .702), p<.001)$ (supporting information Table S5). 


\section{Item Response Theory (IRT) Analyses}

IRT analyses were used to examine the discrimination and threshold parameters of each item, and to obtain item characteristics curves (ICC) to inspect the pattern of item responses, as well as test information curves (TIC).

IRT was performed in IRTPRO using the Graded Response Model (GRM) from Samejima, a useful model for analyzing polytomous data. The maximum marginal likelihood estimation (MML) was used to estimate item parameters. IRT was performed separately for the dimensions identified in the CFA (ensuring unidimensionality). Local independence was examined considering the standardized LD (Local dependence) $\chi^{2}$ statistics for each item pair $(<$ 10) [33]. Item discrimination parameters were examined according to Baker's [34] guidelines: 0.01-0.24=very low discrimination; $0.25-0.64=$ low discrimination; $0.65-1.34=$ moderate discrimination; $1.35-1.69=$ high discrimination; more than $1.70=$ very high discrimination. Threshold parameters were examined according to Toland's guidelines [33], in which ideal threshold should range between -3 and 3. Differential item functioning (DIF) analysis was used to examine item functioning across different groups (age and type of cancer), with a significant chi-squared test indicating differences in items' performance across groups.

Discrimination and threshold parameters of all items are presented in supporting information Table S6. Test information curve (TIC) for the two dimensions of the ERQ is presented in supporting information Figure S1. Local independence was obtained for ES (LD $\chi^{2}$ statistics ranging from 0.8 to 7.1 ) but not for CR (LD $\chi 2$ statistics ranging from 3.6 to 21.5). To determine if this violation of LD assumptions was problematic, item calibrations were conducted without the suspected items. Obtained slopes and threshold parameters were highly similar to the 
slopes and threshold parameters obtained when all items were included. Also, the inspection of residual correlations showed that in both subscales there is local independence for all items (correlation values $<.20$ ) (see supporting information Table S7).

Most items were highly discriminant $\left(\alpha^{1}>1.35\right)$, meaning that they helped discriminate between the two ER constructs. Only items 4 and 5 presented values suggesting moderate discrimination $\left(\alpha^{l}>0.81\right)$. Threshold parameters, which indicate the trait level at which the likelihood of endorsing a given response choice is $50 \%$, ranged from -0.36 to -3.00 for the first parameter, -2.15 to 0.44 for the second parameter, -1.67 to 0.98 for the third parameter, -0.74 to 2.26 for the fourth parameter, -0.36 to 2.67 for the fifth parameter, and 0.19 to 3.36 for the sixth parameter. DIF analyses indicated that there were no significant differences in how items functioned according to the type of cancer. With regard to age, no differences were found, with the exception of item 5 from $\mathrm{CR}\left(\chi^{2}(7)=15.8, p=.03\right)$, which was more discriminant for the younger women. The TIF indicated that the ERQ provides the greatest amount of information for individuals with moderate levels of ES and CR.

\section{Convergent, Concurrent, and Discriminant Validity}

Convergent validity was assessed by estimating two indices, namely the average variance extracted (AVE) (>.50) and the composite reliability (>.70) [35]. The association between ERQ scales and other constructs were examined to assess convergent and concurrent validity.

Discriminant validity was assessed by comparing the intercorrelation of the ES and CR scales to the square root of the AVE of each dimension [35]. 
AVE, square root of the AVE, and composite reliability values provided evidence for the convergent and discriminant validity of ES and $\mathrm{CR}^{2}$ (see supporting information Table S5). Evidence for convergent validity and concurrent validity was also found by examining associations between variables. Correlations between variables are presented in supporting information Table S8.

\section{Discussion}

This study investigated the psychometric properties of the ERQ in a sample of women with cancer. Past research has highlighted the role that ER can play in adapting to these challenges [4]. Modern tools for assessing these regulatory strategies, however, have rarely been employed in research with cancer patients and no studies have examined the validity of these instruments in this context. This study addresses the paucity of research on ER using the ERQ in cancer populations.

Our findings indicate that the ERQ is a promising instrument to assess emotion regulatory strategies in cancer populations. This study provides support for its scale structure, internal consistency, test-retest reliability, and validity among women with cancer. The twofactor structure - CR and ES - proposed by Gross and John [9] and found in numerous studies of non-cancer populations was confirmed [e.g., 36]. The lack of variation in ERQ structure across age and type of cancer adds important evidence for the robustness of this measure and its likely utility in studying psychosocial adaptation to cancer. The CR and ES factors in this study were found to be moderately correlated as they have in a number of other studies [e.g., 12,17] since

\footnotetext{
${ }^{2} \mathrm{CR}$ had an AVE value lower than .50. However, according to Fornell and Larcker [35] an AVE value
} higher than .4 indicates adequate convergent validity if the CP value is higher than .6, which was the case. 
Gross and John [9] first provided evidence of the independence of these factors. The moderate overlap among factors suggests that in the context of meeting the challenges of cancer (and in many other contexts) individuals who tend to reappraise stressful situations in attempt to diminish their negative affect also tend to suppress the expression of negative emotions.

The IRT analyses provide evidence about the functioning of each of the items on the ERQ. The analysis demonstrated that all items achieved a moderate to high level of discrimination in this sample of women. Items did not appear to be redundant or problematic and there is no reason to drop any item from the scale. One item differed in its informativeness across young and old participants - item 5 from CR. No modification to this item was proposed since the degree of difference was small and this is the only item that differed in this way across samples. Future studies, however, should continue to investigate the functioning of this item. The examination of the test information curve (TIC) showed that the maximum amount of information (i.e., measurement precision) for both scales were around the mean of the trait levels. For both scales, the amount of information was less accurate at the lowest and highest levels of the trait. This means that outside of these ranges score estimates are less precise.

The overall reliability of the ERQ is good. Alphas above .70 for both ERQ scales indicate good internal consistency. The values obtained in this study are comparable to those found in previous studies [e.g., 9,15,16] (.68 to .76 for ES;.75 to .82 for CR). A test-retest ICC reliability greater than .50 indicates a moderate to high degree of stability across a six-week period. This stability suggests that the ERQ is capturing a regulatory style that remains relatively consistent across time in women with cancer. IRT analyses indicate that the reliability of both dimensions differs somewhat depending on levels of ES and CR. Overall, the results from IRT analyses 
indicate that all items were moderately to highly discriminant and that items were best suited for identifying moderate levels of ES and CR.

The validity of the ERQ was supported by a number of findings. Medium to large correlations between ES and emotional control, self-efficacy, and alexithymia were found. Consistent with expectations, individuals who report typically suppressing their emotions are more likely to report controlling their emotions and not expressing them to others, and to have a greater difficulty in identifying and describing their emotions. These associations are consistent with other studies [e.g., 37]. Suppressors also tend to report lower emotional self-efficacy, including concerns about difficulties communicating their emotions and staying focused on the present moment. In contrast, CR was positively correlated with emotional self-efficacy. This pattern of findings is consistent with past studies [e.g., 9] in which ES was negatively correlated with sharing emotions with others (both positive and negative) and CR was positively correlated with greater sharing of emotion.

As anticipated, ES was also positively correlated with attachment avoidance. Avoidantly attached individuals tend to adopt strategies to deactivate or diminish attachment or relational concerns or focus, such as denying emotional experiences and suppressing negative emotions [38], CR was not significantly correlated with attachment, which is consistent with previous findings from Gross and John [9].

Both ES and CR were associated with perceived QOL, providing support for the concurrent validity of ERQ and providing evidence for the importance of studying ER in the context of cancer. Individuals who reported using more ES also reported lower QOL across all domains. In contrast, CR was positively correlated with psychological and social QOL. This pattern is consistent with past findings [e.g., 9] in which ES is generally associated more with 
negative indicators of well-being, while $\mathrm{CR}$ is related to more positive outcomes. For individuals with cancer, the adoption of these strategies to regulate emotions appears to have different consequences.

Future research should try to examine these associations more closely to provide more information on the mechanisms underlying these connections and the contexts in which these connections are boosted or minimized. Research in other areas suggests that flexibility in regulatory strategies is important and that even suppressive strategies can be beneficial in certain circumstances or for certain individuals [39]. More research is needed to identify situation-based and person-based moderators of links between ER and well-being.

In addition to a number of strengths, the current study has two main limitations. First, our sample included only women with cancer and the majority of these women were not currently undergoing treatment. Future studies should explore whether the factorial structure found remains valid and invariant across men with cancer and across patients in different phases of the disease. Second, this study had a relatively small sample size for IRT analyzes, which can limit accurate parameter estimates. However, studies have successfully used IRT analysis with smaller samples [e.g., 40].

In summary, this was the first study to rigorously examine the factor structure of the ERQ in cancer and the first to conduct IRT analyses of the ERQ items. The IRT analyses provide support for the discriminant power of each item on both ERQ dimensions and systematically evaluate how respondent characteristics might influence their utility. This study examined the validity of the ERQ using a wide range of measures. Our results also provide evidence that the ERQ is a valuable research and clinical assessment tool of ER in the context of cancer. The results suggest that studying regulatory strategies in the context of cancer is important since there 
are clear links with QOL, an important psychosocial outcome. Given the impact of ER strategies on psychosocial adaptation to cancer, the ERQ can be used as a screening tool for determining the psychosocial support needs of patients with cancer who can benefit from evidence-based interventions targeting ER strategies. 


\section{References}

1. Adler NE, Page AE. Cancer care for the whole patient: Meeting psychosocial health needs. Washington DC: The National Academies Press; 2008.

2. Wang Y, Yi J, He J, Chen G, Li L, Yang Y, Zhu X. Cognitive emotion regulation strategies as predictors of depressive symptoms in women newly diagnosed with breast cancer. Psychooncology. 2014; 23:93-99. doi: http://dx.doi.org/10.1002/pon.3376

3. Porter L, Keefe F, Hurwitz H, Faber M. Disclosure between patients with gastrointestinal cancer and their spouses. Psychooncology. 2005;14:1030-1042. doi: http://dx.doi.org/10.1002/pon.915

4. Brandão T, Tavares R, Schulz MS, Matos PM. Measuring emotion regulation and emotional expression in women with breast cancer: a systematic review. Clin Psychol Rev. 2015;43:114-127. doi: 10.1016/j.cpr.2015.10.002

5. Gross JJ. Antecedent- and response-focused emotion regulation: Divergent consequences for experience, expression, and physiology. J Pers Soc Psychol. 1998;74: 224-237. doi: http://dx.doi.org/10.1037/0022-3514.74.1.224

6. Schulz MS, Lazarus RS. Emotion regulation during adolescence: A cognitive mediational conceptualization. In Kerig PK, Schulz MS, Hauser ST, eds. Adolescence and beyond: Family processes and development. Oxford: University Press; 2012: 19-42.

7. Waldinger RJ, Schulz MS. Facing the music or burying our heads in the sand?: Adaptive emotion regulation in midlife and late life. Res Hum Dev. 2010;7: 292-306. doi: http://dx.doi.org/10.1080/15427609.2010.526527 
8. Dixon-Gordona KL, Aldao A, Reyes A. Emotion regulation in context: Examining the spontaneous use of strategies across emotional intensity and type emotion. Pers Indiv Differ. 2015;86: 271-276. doi: http://dx.doi.org/10.1016/j.paid.2015.06.011

9. Gross JJ, John OP. Individual differences in two emotion regulation processes: Implications for affect, relationships, and well-being. J Pers Soc Psychol. 2003;85: 348-362. doi: http://dx.doi.org/10.1037/0022-3514.85.2.348

10. Sheppes G, Gross J. Emotion regulation effectiveness: What works when. In Tennen HA, Suls JM, eds. Handbook of psychology (vol 5): Personality and Social Psychology. New York: Wiley; 2012:391-406.

11. Spaapen DL, Waters F, Brummer L, Stopa L, Bucks RS . The Emotion Regulation Questionnaire: Validation of the ERQ-9 in two community samples. Psychol Assessment. 2014;26:46-54. doi: http://dx.doi.org/10.1037/a0034474

12. Uphill MA, Lane AM, Jones MV. Emotion Regulation Questionnaire for use with athletes. Psychol Sport Exerc, 2012;13:761-770. doi: http://dx.doi.org/10.1016/j.psychsport.2012.05.001

13. Matsumoto D, Yoo SH, Nakagawa S, Alexandre J, Altarriba J, Anguas-Wong AM . . . Zengeya A. Culture, emotion regulation, and adjustment. J Pers Soc Psychol. 2008;94: 925-937. doi: http://dx.doi.org/10.1037/0022-3514.94.6.925

14. Wiltink J, Glaesmer H, Canterino M, Wölfling K, Knebel A, Kessler H, . . Beutler ME . Regulation of emotions in the community: Suppression and reappraisal strategies and its psychometric properties. Psychosoc Med, 2001:8. doi: 10.3205/psm000078 
15. Balzarotti S, John OP, Gross JJ. An Italian adaptation of the Emotion Regulation Questionnaire. Eur J Psychol Assess, 2010;26:61-67. doi: http://dx.doi.org/10.1027/1015-5759/a000009

16. Cabello R, Salguero JM, Fernández-Berrocal1 P, Gross JJ. A Spanish adaptation of the Emotion Regulation Questionnaire. Eur J Psychol Assess, 2013;9:234-240. doi: http://dx.doi.org/10.1027/1015-5759/a000150

17. Gómez-Ortiz O, Romera EM, Ortega-Ruiz R, Cabello R, Fenández-Berrocal P. Analysis of emotion regulation strategies in Spanish adolescents: Validation of the Emotion Regulation Questionnaire. Front Psychol. 2016;7:1959. doi: 10.3389/fpsyg.2015.01959

18. Watson M, Greer S. Development of a questionnaire measure of emotional control. $J$ Psychosom Res. 1983;27: 299-305. doi: http://dx.doi.org/10.1016/0022-3999(83)90052-1

19. Patrão I. O ciclo psico-oncológico no cancro da mama: Estudo do impacto psicossocial do diagnóstico e dos tratamentos [The psycho-oncology cycle of the breast cancer: Psychosocial impact of diagnosis and treatmens] (Unpublished doctoral dissertation). Universidade Nova de Lisboa, Lisboa; 2007.

20. Giese-Davis J, Koopman C, Butler LD, Joss J, Classen C, Roberts J ... Spiegel D. The Stanford Emotional Self-Efficacy Scale-Cancer: Reliability, validity, and generalizability. In Nyklícek I, Temoshok L, Vingerhoets A, eds. Emotional expression and health: Advances in theory, assessment and clinical applications. New York: Brunner-Routledge; 2004:204-222.

21. Brandão T, Matos PM. Portuguese version of the Stanford Emotional Self-Efficacy Scale Cancer. Unpublished manuscript. 
22. Bagby RM, Parker JDA, Taylor GJ. The twenty-item Toronto Alexithymia Scale-I. Item selection and cross-validation of the factor structure. J Psychosom Res. 1994;38:23-32. doi: http://dx.doi.org/10.1016/0022-3999(94)90005-1

23. Prazeres N, Taylor GJ, Parker JDA. Escala de Alexitimia de Toronto de Vinte Itens (TAS20) [Toronto Alexithymia Scale of 20 item]. In Almeida LS, Simões MR, Machado M, Gonçalves MM, eds. Avaliação psicológica: Instrumentos validados para a população portuguesa [Psychological assessment: Validated instruments to the portuguese population]. Coimbra: Quarteto; 2008:95-107.

24. Fraley RC, Heffernan ME, Vicary AM, Brumbaugh CC. The experiences in close relationships-relationship structures questionnaire: A method for assessing attachment orientations across relationships. Psychol Assessment. 2011;23: 615-625. doi: http://dx.doi.org/10.1037/a0022898

25. Moreira H, Martins T, Gouveia MJ, Canavarro MC. Assessing adult attachment across different contexts: Validation of the Portuguese version of the Experiences in Close Relationships - Relationship Structures Questionnaire. J Pers Assess. 2015;97:22-30. doi: http://dx.doi.org/10.1080/00223891.2014.950377

26. Vaz Serra A, Canavarro MC, Simões MR, Pereira M, Gameiro S, Quartilho M J, ... Paredes T. Estudos psicométricos do instrumento de avaliação da qualidade de vida da Organização Mundial de Saúde (WHOQOL-Bref) para Português de Portugal [Psychometric properties of The World Health Organization quality of life assessment (WHOQOL-Bref) to Portuguese of Portugal]. Psiquiatria Clínica. 2006; 27: 41-49. 
27. Cai L, Thissen D, du Toit SHC. IRTPRO: Flexible, multidimensional, multiple categorical IRT modeling [Computer software]. Lincolnwood, IL: Scientific Software International;2011.

28. Kim HY. Statistical notes for clinical researchers: Assessing normal distribution (2) using skewness and kurtosis. Restor Dent Endod. 2013;38:52-54. doi: http://dx.doi.org/10.5395/rde.2013.38.1.52

29. Hooper D, Coughlan J, Mullen MR. Structural equation modeling: Guidelines for determining model fit. EJBRM. 2008;6:53-60.

30. Gullone E, Taffe J. The Emotion Regulation Questionnaire for children and adolescents (ERQ-CA): A psychometric evaluation. Psychol Assess. 2014;24:409-417.doi: http://dx.doi.org/10.1037/a0025777

31. Azim HA, Partridge AH. Biology of breast cancer in young women. Breast Cancer Res. 2014;16: 427. doi: 10.1186/s13058-014-0427-5

32. Hair J, Black W, Babin B, Anderson R. Multivariate data analysis (7th Ed.). Upper Saddle River, NJ: Prentice-Hall; 2010.

33. Toland MD. Practical guide to conducting an item response theory analysis. J Early Adolesc. 2014;34:120-151. doi: http://dx.doi.org/10.1177/0272431613511332

34. Baker FB. The basics of item response theory (2nd Ed.). College Park, MD: Eric Clearing House on Assessment and Evaluation; 2001.

35. Fornell C, Larker DF. Evaluating structural equation models with unobservable variables and measurement error. J Mark Res. 1981;18:39-50. 
36. Batistoni S S T, Ordonez T N, Silva T B L D, Nascimento P P P D, Cachioni M. Emotional Regulation Questionnaire (ERQ): Psychometric indicators and affective relations in an elderly sample. Psicologia: Reflexão e Crítica. 2013;26:10-18.

37. Laloyaux J, Fantini C, Lemaire M, Luminet O, Larøi F. Evidence of contrasting patterns for suppression and reappraisal emotion regulation strategies in alexithymia. J Nerv Ment Dis. 2015;203:709-718. doi: http://dx.doi.org/10.1097/NMD.0000000000000353

38. Mikulincer M, Shaver PR. Attachment in adulthood: Structure, dynamics, and change ( $2^{\text {nd }}$ Ed). New York: Guilford Press; 2016.

39. Bonanno GA, Burton CL. Regulatory flexibility: An individual differences perspective on coping and emotion regulation. Perspect Psychol Scienc. 2013;8:591-612. Doi: http://dx.doi.org/10.1177/1745691613504116

40. Zaidman-Zait, A., Mirenda, P., Zumbo, B. D., Wellington, S., Dua, V., \& Kalynchuk, K. (2010). An item response theory analysis of the Parenting Stress Index - Short Form with parents of children with autism spectrum disorders. J Child Psychol Psychiatry; 51:12691277. doi: $10.1111 / \mathrm{j} .1469-7610.2010 .02266 . x$ 